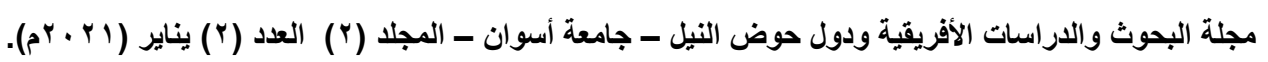

أثر التعليم قبل الجامعى على النمو الاقتصادى فى أفريقيا

"حالة التعليم ما قبل الجامعى فى أفريقيا"

$$
\text { الباحث }
$$

حمد عويض إبراهيم عويض

أ.د. مكادى عبد المجيا سليمان

استاذ الاقتصاد الزراعى، جامعة المنيا

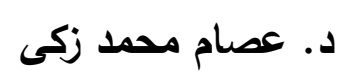

معهد بحوث الاقتصاد الزراعى مركز البحوث الزراعية 
الملخص

إستهدف البحث بصفة أساسية دراسة وتوضيح تأثير التعليم والتدريب على إقتصاد الدول الأفريقية، وكذا إلقاء الضوء على العلاقة بين التعليم والاقتصاد والتتمية كعلاقة تبادلية حيث يسهم التعليم في التنمية بشكل مباشر، وإستعرض البحث إستراتيجية التعليم القارية لأفريقيا والتى تتضمن مجموعة من الأهداف الاستراتيجية تتمثل فى إثنى عشر هدف تم إستعراضهم بمتن البحث.

كلمات مفتاحية: التعليم قبل الجامعى، النمو الاقتصادى، حالة التعليم في إفريقيا. مقدمة: يمثل التعليم القلب النابض للأمم، كما أنه في السنوات الأخيرة يظهر التفوق العالمي جليًا في مدى المنافسة في تطوير نظم التعليم للدول المتقدمة، وتحدد المراحل التعليمية الغايات التربوية والتعليمية من خلال المدرسة معالم الطريق لمواكبة العصر الحالى وتحقيق أهداف المجتمع، كما أن العلاقة وطيدة بين التعليم الذي هو اساس المعرفة والنمو الاقتصادي، لكن السؤال الذي يطرح نفسه وهو ماهي العلاقة بين التعليم الجيد والنمو الاقتصادي، أو جودة التعليم والنمو الاقتصادي؟' لايكاد يمر علينا يوم الا ونسمع حديثاً يدور عن ضرورة وجود تعليم جيد يسمح للدول بالازدهار ' عبدالله ونيس الترهونى (دكتور)، العلاقة بين النمو الاقتصادى وجودة التعليم، بوابة أفريقيا

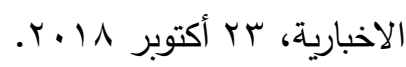


والتقدم، بل وأعتبره البعض من المسلمات اليومية، وقد أظهرت دراسة لجامعة "هارفارد" إلى أن أهمية التعليم وتأثيره في النمو يرجع لأكثر من سبب، على رأسها مساهمة رأس المال البشري الذي يقدر بنسبة rج \% من الناتج الاجمالي العالمي، إلى جانب زيادة نسبة الخدمات في اجمالي الناتج حول العالم، بينما تقدم الآلات والموارد الطبيعية حازت على ^^٪ المتبقية، وعلى جانب آخر فقد خلصت إحدى دراسات البنك الدولي حول تأثير التعليم على الدخل الى أنه "في مقابل كل عام يقضيه الانسان في الدراسة بعد المرحلة الابتدائية فان دخله سيزيد بنسبة ـ ( سنويًا من تاريخ التحاقه بالعمل" هذا على جانب الفرد، أما من جانب الدولة أو المجتمع وبحسب نفس الدراسة والتي تمت باستخدام إختبارات عالمية في الرياضيات والعلوم ومدى علاقتها بمستوى نمو الدخل القومي فقد خلصت الدراسة التي أجُريت على بيانات ــ دولة وعلى مدار • ــ عامًا متواصلة إلى تفوق الدول التي تتمتع بتعليم أفضل (أجود) على تلك التي لا تتمتع به في معدلات النمو بنسبة بلغت r\% سنويًا، وبعبارة أوضح فكل دولار يتم إنفاقه على التعليم في أية دولة لا سيما الدول النامية يعود إليها بدخلًا سنويًا بين V - - . دولارات وفي فترة من 10 الى • r سنة، وهذا مؤشر واضح بأن الاستثمار في الانسان رابح 
ولكن على المدى الطويل'، وأن هذا الاستثمار ليس مجرد تحسين لمهارات وقدرات الأفراد أو الثعوب في حينها ثم تضدحل وتتتهي.

ويختلف تأثير التعليم الجيد بين الدول المتقدمة ونظيراتها النامية، ففي الأولى لايتعدى تأثير التفاوت في جودة التعليم على معدلات النمو الاقتصادي ه. • \% سنويًا وذلك بسبب تدني معدلات النمو رغم ثباتها أولاً، ولتشابه أسس النظم التعليمية ثانيًا، أما في الدول النامية فيصل تأثير جودة التعليم إلى فارق نمو يزيد عن ب٪ سنويًا، بل ويمكن اعتباره أكبر من ذلك بكثير إذا ما أخذنا في الاعتبار التأثير التراكمي لبرامج التعليم والتي لا يظهر تأثيرها بين ليلة وضحاها كما أشرنا. كما إهتمت العديد من الدراسات والتقارير بحالة التعليم فى أفريقيا ومن تلك الدراسات "مواجهة المستقبل: التعليم من أجل التعلم في إفريقيا"2 الذى إهتمت بوضع مجموعة من السياسات وإلإجراءات التنفيذية اللازمة لبلدان المنطقة لمواجهة التحدي المتمثل في تحسين التعلم مع توسيع نطاق الوصول إلى التعليم الأساسي وإتمامه للجميع.

خلاصة القول أن الاستثمار في الانسان هو افضل استثمار على الاطلاق وإن كانت نتائجه لن تظهر على المدى القصير، وقد أعد البنك الدولي "وصفة" للدول ' عبدالله ونيس الترهونى (دكتور)، العلاقة بين النمو الاقتصادى وجودة التعليم، المرجع السابق. ${ }^{2}$ Facing Forward: Schooling for Learning in Africa, worldbank, March 5, 2018. 
الراغبة في تحقيق نهضة مستقبلية وسريعة عن طريق التعليم والمعرفة وذلك من خلال التركيز على 0 مهارات ((متعلقة بالتتمية البشرية)) وأنها المفتاح الرئيسي للمهارات الإدراكية التي يُشكل تعلمها وإتقانها الخطوة الفارقة في مسيرة تحول الدول من نامية إلى متقدمة وهي: "المهارات الاجتماعية، حل المثكلات، إدارة الوقت والمهارات الشخصية، مهارات الاتصال، ومهارات التعلم المستمر"'.

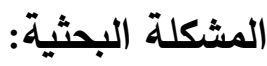
على الرغم من إهتمام العديد من الدراسات والتقارير بحالة التعليم فى أفريقيا وذلك من خلال وضع مجموعة من السياسات وإلإجراءات التنفيذية اللازمة لبلدان المنطقة لمواجهة التحدي المتمثل في تحسين التعلم مع توسيع نطاق الوصول إلى التعليم الأساسي وإتمامه للجميع، إلا أنه كان نظام التعليم الموروث في غالبية الدول الإفريقية نظاماً استعماريّاً، هدفه الأساسي إنثاء قوة عمل تعتمد على الاستعماريين في روح المبادرة والإبداع، وتخدم مصالحهم وتلبّي احتياجاتهم في الدول التي سيطروا عليها' , مما أدى- حتى بعد مغادرتهم المزعومة- إلى فقدان التعليم في العديد من دول القارة للروح الإقريقية الأصيلة، التي تهدف إلى منح الطلاب المهارات والمعرفة التي تجعلهم أعضاءً متكاملين في مجتمعاتهم المحلية, يستطيعون بناء قدراتهم وتسخير

' عبدالله ونيس الترهونى (دكتور)، العلاقة بين النمو الاقتصادى وجودة التعليم، مرجع سابق. ${ }^{2}$ Nwanosike, O. F \& ,.Onyije, F, Colonialism and education. Mediterranean journal of social sciences, $2(4), r \cdot 11$. 
مواردهم لتتمية البنى التحتية والاستجابة للاحتياجات الاجتماعية، وقد سقطت الأقنعة عن حقيقة هذه الأنظمة التعليمية الموروثة بعد أكثر من نصف قرن- أو عقود- من استقلال الدول الإفريقية, وكثفت مختلف التحديات ومطالب المجتمع الإفريقي والعالم التنافسي، نقاط ضعفها؛ في ظلّ الظروف المتغيرة في القرن الحادي والعشرين (العصر المعلوماتي والرقمي) الذي تصاحبه الأزمات الجديدة والمتجدة, ليكون من الضروري تصميم نوعٍ جديدٍ من التعليم، أو تطويره تطويراً شاملاً ليتناسب مع تاريخ الدول الإقريقية وليتجاوب مع ظروف التحديات الراهنة. هدف البحث: يستهف البحث بصفة أساسية دراسة وتوضيح تأثير التعليم والتدريب على إقتصاد الدول الأفريقية، وكذا إلقاء الضوء على العلاقة بين التعليم والاقتصاد والتتمية كعلاقة تبادلية حيث يسهم التعليم في التتمية بشكل مباشر من خلال ما يقدمه من أيدى عاملة مؤهلة ومدربة (رأس مال بشري) علاوة على ما يغرسه التعليم من قيم ومبادئ تجاه سوق العمل، ومن ناحية أخرى فإن الاقتصاد يقدم للتعليم موارده المختلفة، ومن هنا يمكن القول ان التعليم هو حلقة الوصل بين رأس المال البشري والتتمية الاقتصادية أخذا في الاعتبار العلاقة التبادلية من كافة أطراف المعادلة التى يمكن اعتبار النجاح

${ }^{1}$ Masemula M.B, Whose Knowledge is Transmitted through Public Education in Africa?. In: Abdi A.A., Shultz L., Pillay T. eds Decolonizing Global Citizenship Education, 2015. 
في توازن العلاقة بين متغيراتها الثلاثة (التعليم- رأس المال البشري- التنمية الاقتصادية) يضمن لأى دولة تحقيق العديد من النتائج الإيجابية على المستويين

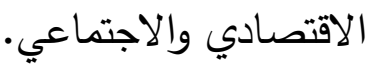
الاسلوب البحثى ومصادر البيانات: تحقيقـا لهـدف البحــث اسـتُخدمت أسـاليب الإحصــاء الوصـفي للمتغيـرات الاقتصادية محل الدراسة، وقد تطلب لإجراء ولتنفيذ البحث الاستعانه بمختلف البيانات الإحصـائية الثانويـة المنشـورة والتـي تصـدرها العديـد مـن الجهات على مواقـع شبكة المعلومـات الدوليـة (إنترنتـ)، وكذا تقارير البنك الدولى، ومنظمـة اليونسكو، منظمـة العمل الدولية، بالاضافة للاستعانة بالعديد من الدراسات والتقارير والبحوث ذات الصلة بموضوع البحث.

\section{المناقثة البحثية}

إهتمت العديد من الدراسات والتقارير بحالة التعليم فى أفريقيا ومن تلك

الدراسات "مواجهة المستقبل: التعليم من أجل التعلم في إفريقيا"1 والذى إهتمت بوضع مجموعة من السياسات والإجراءات التنفيذية اللازمة لبلدان المنطقة لمواجهة التحدي المتمثل في تحسين التعلم مع توسيع نطاق الوصول إلى التعليم الأساسي وإتمامه للجميع، وأكدت الدراسة على أهمية مواءمة نظام التعليم بحيث يركز على نتائج التعلم

${ }^{1}$ Facing Forward: Schooling for Learning in Africa, worldbank, op. 
وضمان حصول جميع الأطفال على مدارس جيدة ومواد تعليمية جيدة ومعلمين جيدين، كما أنه وفقًا للتحديات التي واجهت تلك البلدان الأفريقية في التسعينيات والتقدم التعليمي الذي حقتته على مدار الـ هب عامًا الماضية، مما يسمح لبلدان المنطقة بالتعلم من بعضها البعض، كما تستخلص الدراسة دروسًا حول "ما يصلح" لتعزيز التعلم وما هو مطلوب من أجل تتفيذ أفضل وحددت خطوات ملموسة في أربعة مجالات ذات أولوية تتمثل فى:

1- توفير التعليم الأساسي الشامل مع التركيز على الوصول العادل. r - ب والجودة والاستمرار ضمان الإدارة الفعالة. r- ودعم المعلمين وزيادة التمويل للتعليم الجيد. ع- وتعزيز القدرة المؤسسية.

كما أثارت إلى تأثر التقدم التعليمي المستقبلي بمعلات الخصوبة المتوقعة والنمو الاقتصادي بتلك الدول الأفريقية. حيث أنه طبيعة العلاقة بين التعليم والاقتصاد والتتمية هي علاقة تبادلية حيث يسهم التعليم في التتمية بشكل مباشر من خلال ما يقدمه من أيدى عاملة مؤهلة ومدربة (رأس مال بشري) علاوة على ما يغرسه التعليم من قيم ومبادئ تجاه سوق العمل، ومن ناحية أخرى فإن الاقتصاد يقدم للتعليم موارده المختلفة، ومن هنا يمكن 
القول ان التعليم هو حلقة الوصل بين رأس المال البشري والتتمية الاقتصادية أخذا في الاعتبار العلاقة التبادلية من كافة أطراف المعادلة التى يمكن اعتبار النجاح في توازن العلاقة بين متغيراتها الثلاثة (التعليم- رأس المال البشري- التتمية الاقتصادية) يضمن لأى دولة تحقيق العديد من النتائج الإيجابية على المستويين الاقتصادي والاجتماعي. تأثير التعليم والتدريب على اقتصاد الدول ركزت العديد من البلدان على تطوير نظام تعليمي قادر على إنتاج عمال قادرين على العمل في صناعات جديدة، مثل تلك الموجودة في ميادين التكنولوجيا والعلوم، ويعود هذا جزئيا إلى أن الصناعات القديمة في الاقتصادات المتقدمة أصبحت أقل قدرة على المنافسة، وبالتالي كانت احتمالية استمرار سيطرتها على المشهد الصناعي أقل، أيضا فقد ظهرت حركة لتحسين التعليم الأساسي للسكان، مع اعتقاد متزايد بأن جميع الناس لديهم الحق في التعليم. عندما يتحدث الاقتصاديون عن "التعليم"، فإن التركيز لا يقتصر على العمال الذين يحصلون على شهادات جامعية، فغالبًا ما يتم تقسيم التعليم إلى مستويات محددة وهي' : الابتدائية، والمدرسة الإعدادية والثانوية، ومرحلة ما بعد الثانوية أي الجامعة والمدارس المهنية، ويصبح اقتصاد أي بلد أكثر إنتاجية مع زيادة نسبة العمال المتعلمين لأن العاملين المتعلمين يمكنهم القيام بمزيد من الفعالية بمهام تتطلب معرفة

${ }^{1}$ https://www.almrsal.com/post/767576. 
القراءة والكتابة والتتكير النقدي، ومع ذلك فإن الحصول على مستوى أعلى من التعليم ينطوي أيضًا على تكلفة، ولكن قد لا تحتاج البلد إلى توفير شبكة واسعة من الكليات أو الجامعات للاستفادة من التعليم ؛ حيث يمكنها توفير برامج محو الأمية الأساسية لكي ترى تحسينات اقتصادية، فالبلدان التي لديها نسبة أكبر من سكانها الذين يتدربون ويتخرجون من المدارس، تثهذ نمواً اقتصادياً أسرع من البلدان ذات العمال الأقل تعليماً، ونتيجة لذلك يوفر العديد من البلدان التمويل للتعليم الابتدائي والثانوي لتحسين الأداء الاقتصادي، وبهذا المعنى يعتبر التعليم استثمارًا في رأس المال البشري، على غرار الاستثمار في معدات أفضل. ووفقاً لليونسكو وبرنامج الأمم المتحدة للتنمية البشرية، فإن نسبة عدد الأطفال الذين بلغوا سن الالتحاق بالمدارس الثانوية الرسمية الملتحقين بالمدارس هي نسبة عالية في الدول المتقدمة أكثر من الدول النامية، ويختلف هذا عن الإنفاق على التعليم كنسبة مئوية من الناتج المحلي الإجمالي، والذي لا يرتبط دائمًا بقوة بمدى تعليم سكان بلد ما، ولذلك فإن البلد التي تنفق نسبة عالية من الناتج المحلي الإجمالي على التعليم لا يجعل بالضرورة سكان البلاد أكثر تعليما. كذلك يمكن للتدريب أن يجعل أجر العامل أكبر فأصحاب العمل يدفعون أجورا أكثر عندما تتطلب المهام المطلوبة لإكمال وظيفة مستوى تعليم أعلى وهكذا في حين 
أنه قد يتم تخفيض الأجر على المدى القصير لحين انتهاء العامل من التدريب إلا أنه من المرجح أن تكون الأجور أعلى في المستقبل بمجرد اكتمال التدريب. كان نظام التعليم الموروث في غالبية الدول الإفريقية نظاماً استعماريّا، هدفه الأساسي إنشاء قوة عمل تعتمد على الاستعماريين في روح المبادرة والإبداع، وتخدم مصالحهم وتلبّي احتياجاتهم في الدول التي سيطروا عليها ', مما أدى- حتى بعد مغادرتهم المزعومة- إلى فقدان التعليم في العديد من دول القارة للروح الإقريقية الأصيلة، التي تهدف إلى منح الطلاب المهارات والمعرفة التي تجعلهم أعضاءً متكاملين في مجتمعاتهم المحلية, يستطيعون بناء قدراتهم وتسخير مواردهم لتنمية البنى التحتية والاستجابة للاحتياجات الاجتماعية2، وقد سقطت الأقنعة عن حقيقة هذه الأنظمة التعليمية الموروثة بعد أكثر من نصف قرن- أو عقود- من استقلال الدول الإفريقية, وكثفت مختلف التحديات ومطالب المجتمع الإفريقي والعالم التنافسي، نقاط ضعفها؛ في ظلّ الظروف المتغيرة في القرن الحادي والعشرين (العصر المعلوماتي والرقمي) الذي تصاحبه الأزمات الجديدة والمتجدة, ليكون من الضروري تصميم نوعٍ

${ }^{1}$ Nwanosike, O. F \& ,.Onyije, F, Colonialism and education. Mediterranean journal of social sciences, $2(4), r \cdot 11$.

${ }^{2}$ Masemula M.B, Whose Knowledge is Transmitted through Public Education in Africa?,op. 
جديدٍ من التعليم، أو تطويره تطويراً شاملاً ليتناسب مع تاريخ الدول الإقريقية وليتجاوب مع ظروف التحديات الراهنة.

وقد سجّل الدكتور 》كوفي بوسيا《, رئيس وزراء غانا من 1979 إلى بVY 19

اعترافه بفشل نموذج التعليم الاستعماري في بلاده- قبل سلسلة الإصلاحات التعليمية التي شهتها غانا حتى عام V . . r- حيث قال: "في نهاية سنتي الأولى في المدرسة الثانوية في كيب كوست/غانا، عُدْتُ إلى البيت في بلدية وينتشي Wenchi لقضاء عطلة عيد الميلاد، لم أزُر المنزل لمدة أربع سنوات وفي تلك الزيارة أصبحتُ على علمٍ بألم عزلتي ولقد فهمتُ مجتمعنا أقلّ بكثير من الأولاد في عمري الذين لم يذهبوا قطّ إلى المدرسة، وعلى مرّ السنين بعدما التحقتُ بالكلية ثم الجامعة شعرتُ بشكلٍ متزايدٍ أن التعليم الذي تلقيتُه علّمني أكثر وأكثر عن أوروبا، وبشكلٍ أقلّ وأقلّ عن مجتمعي الخاص!'، فليس من المستغرب إذاً أن تتزايد الدعوات إلى التطوير والإصلاح المتمثل في المطالبة بأن تُولي السياسات الوطنية تزويد الطلاب بالمهارات اللازمة اهتماماتها؛ لتلبية احتياجاتهم ومواجهة المشكلات والقضايا المحلية بدلاً من الاهتمام المعهود باكتساب المعرفة التي غالباً ما تثبت عجزها في معالجة القضايا الاجتماعية والتتموية الوطنية".

${ }^{1}$ Rodney, W. How europe underdeveloped Africa. Pambazuka Press, 2012. 
كما أنه في نهاية عام Y V . قدّر المكتب الوطني النيجيري للإحصاء نسبة

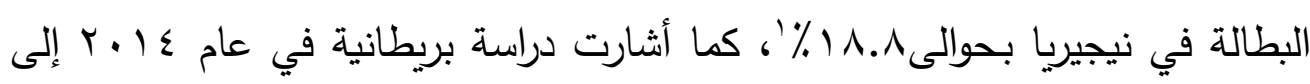
أنّ مدة البحث والحصول على الوظيفة في كينيا قد تستغرق حوالي ه سنوات في المتوسط'·.

أما قادة الأعمال ورؤساء المؤسسات بإفريقيا فدائماً ما يقولون: إنّ هناك وظائف لكن النقص في المواهب الماهرة لأدائها، ويفسر البعض ذلك بأنّ للقيود المالية و"رأس المال البشري" والبنية التحتية تأثيراً سلبيّاً على نطاق المهارات التي يكتسبها الطلاب ونوعيتها, وأنّ هناك فجوةً شاسعةً بين ما تقدّمه المؤسسات التعليمية لطلابها

$$
\text { والمهارات المطلوبة في السوق`؟ }
$$

وما سبق وبعيداً عن مجرّد إعادة تصميم المناهج أو تعديلها يؤكد دعاة

الإصلاح التعليمي أنّ التحوّل الناجح من التعلّم القائم على المعرفة- وهو ما يوجد في عددٍ من الدول الإفريقية بما فيها نيجيريا- إلى التعليم القائم على الكفاءة المحلية

${ }^{1}$ National Bureau of Statistics (2017),"- Labor Force Statistics Vol 1: Unemployment and Underemployment Report", available for download at:http://nigerianstat.gov.ng/download/694 accessed on 19 July, 2018.

${ }^{2}$ British Council (2014), "Can higher education solve Africa's job crisis? Understanding graduate employability in SubSaharan Africa, " available for download at: https://goo.gl/JEdTuj accessed on 19 July 2018.

${ }^{3}$ Quartz Africa (2016). University degrees are not the answer for Africa's unemployed youth, Available online https://goo.gl/gN6f39 accessed on 20 July, 2018. 
ومتطلبات الحلّ للأزمات والتحديات الحالية, يتطلب سياسات وطنية جدّية، وإدماج التكنولوجيا في نُظم التعليم، وزيادة مشاركة المعلمين وأولياء الأمور والقطاع الخاص في عملية صنع القرار ، وتطبيق الإصلاحات التعليمية.

إستراتيجية التعليم القارية لأفريقيا' تتضمن الاستراتيجية مجموعة من الأهداف الاستراتيجية تتمثل فى الأتى: 1- الهدف الاستراتيجي الأول: تنشيط مهنة التدريس لضمان الجودة والملاعمة في جميع مستويات التعليم. r- الهدف الاستراتيجي الثانى: بناء وتأهيل وصيانة وتطوير البنية التحتية للتعليم والسياسات التي تضمن تعلمًا دائمًا وصحيًا ومفيدًا للبيئة في جميع القطاعات الفرعية وللجميع، من أجل توسيع الوصول إلى جودة التعليم. ץ- الهُف الاستراتيجي الثالث: تشخير قدرة تكنولوجيا المعلومات والاتصالات لتحسين الوصول والجودة وإدارة نظم التعليم والتدريب. ع- الهدف الاستراتيجي الرابع: ضمان اكتساب المعارف والمهارات المطلوبة وكذلك تحسين معدلات الإنجاز على جميع المستويات والمجموعات من خلال التنسيق عبر جميع المستويات من أجل التكامل الوطني والإقليمي.

${ }^{1}$ AFRICAN UNION, Continental Education Strategy for Africa, Indicators Manual, 2016-2025. 
ه- الهدف الاستراتيجي الخامس: تسريع العمليات المؤدية إلى المساواة والإنصاف بين الجنسين. צ- الهُف الاستراتيجي السادس: إطلاق برامج محو الأمية الثاملة والفعالة في جميع أنحاء القارة لاستئصال بلاء الأمية. - الهُف الاستراتيجي السابع: تقوية مناهج العلوم والرياضيات في تدريب الثباب ونشر المعرفة والثقافة العلمية في المجتمع. ᄉ- الهذف الاستراتيجي الثامن: توسيع فرص التعليم والتدريب التقني والمهني على المستويين الثانوي والجامعي وتقوية الروابط بين عالم العمل والتعليم والتدريب الأنظمة. 9- الهدف الاستراتيجي التاسع: تتشيط وتوسيع نطاق التعليم العالي والبحث والابتكار لمواجهة التحديات القارية وتعزيز القدرة التنافسية العالمية. • 1- الههف الاستراتيجي العاشر: تعزيز تعليم السلام ومنع النزاعات وحلها في جميع مراحل التعليم ولجميع الفئات العمرية. 11- الههف الاستراتيجي الحادى عثر: تحسين إدارة نظام التعليم وكذلك البناء والتتبيت تعزيز القدرة على جمع البيانات وإدارتها وتحليلها التواصل والاستخدام. r ا - الههف الاستراتيجي الثانى عشر : تشكيل تحالف من أصحاب المصلحة لتسهيل

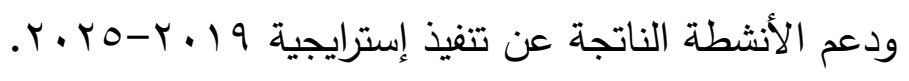


- المؤشرات التى تعكس أثر التعليم على النمو الاقتصادى بأفربقيا أ- معدل النمو السنوي للناتج لكل عامل

يظهر هذا المؤشر معدلات النمو السنوية لإنتاجية العمل، ويمثل النمو فى إنتاجية وحدة العمل أى المنتج لكل وحدة عمل (مقاسة من حيث عدد الأشخاص العاملين) خلال فترة زمنية معينة، ويسح هذا المؤشر بتقييم معدلات النمو فى انتاجية العمالة المساهمة فى الناتج المحلى الاجمالى بمرور الوقت، وبالتالي توفير معلومات حول كفاءة وجودة رأس المال البثري في عملية الإنتاج لوضع اقتصادي واجتماعي معين، بالاضافة لذلك المدخلات التكميلية الأخرى والابتكارات المستخدمة في الإنتاج، يتبين من جدول (1) إنتاجية وحدة العمل بالعالم وبأفريقيا خلال الفترة (• •. (-

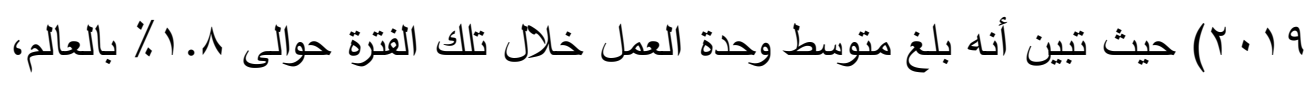
فى حين بلغ حوالى 9 . . ل لكل عامل بأفريقيا أى ما يساوى نصف معدل الناتج لوحدة العمل على مستوى العالم، ويتسم معدل نمو إنتاجية العامل بأفريقيا خلال تلك الفترة

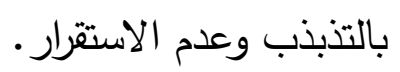

ب- معدل فقر العمالة (النسبة المئوية للعاملين الذين يعيثون بأقل من .9.9 دولار أمريكي) 
يبين هذا المعدل النسبة المئوية للأشخاص العاملين الذين يعيشون في فقر

بالرغم من أنهم يعملون، ويتم تعريف الفقر باستخدام خط الفقر الدولي البالغ .9.9. دولار أمريكي في اليوم، أى النسبة المئوية من العاملون الذين يتقاضون أقل من 9. دولار يوميا مقابل أدائهم لمهام وظائفهم، يتبين من جدول (Y) نسب الفقر بين العمالة فى الفئات العمرية المختلفة التى فى سن العمل بالعالم وبأفريقيا والتى يتبين منها إنخفاض تلك النسب بمرور الزمن على مستوى النوع (رجال، نساء)، وكذا على مستوى الفئات العمرية المختلفة القادرة على العمل والمشاركة فيه وهذا يبين أنه كلما زاد مستوى التعليم والالمام بالمعرفة والتكنولوجيا كلما كانت هناك وظائف أفضل وكذا الأجور أو المرتبات أفضل وبالتالى تتحسن مستويات المعيشة. جدول (1) معدل النمو السنوي للناتج المحلي الإجمالي الحقيقي لكل شخص عامل (الناتج المحلي

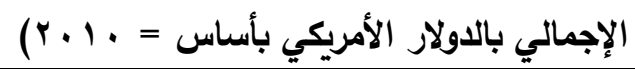

\begin{tabular}{|c|c|c|}
\hline أفربقيا & العالم & السنة \\
\hline 3.0 & 3.3 & 2010 \\
\hline-0.6 & 2.1 & 2011 \\
\hline 4.0 & 1.4 & 2012 \\
\hline 1.5 & 1.6 & 2013 \\
\hline 1.3 & 1.6 & 2014 \\
\hline 0.4 & 1.6 & 2015 \\
\hline-0.6 & 1.4 & 2016 \\
\hline 0.5 & 1.9 & 2017 \\
\hline-0.1 & 1.6 & 2018 \\
\hline-0.5 & 1.4 & 2019 \\
\hline 0.9 & 1.8 & المتوسط \\
\hline
\end{tabular}


$\underline{\text { Source }}$ :International Labour Organization, Statistics and database, labor market indicators.

ج- نسبة الشباب (الذين تتراوح أعمارهم بين 0 (1-؟ ب عامًا) غير الملتحقين بالتعليم أو العمل أو التدربب

يشير إلى نسبة الثباب غير الملتحقين بالتعليم أو العمل أو التدريب المعروف باسم معدل "NEET" أى عدد الثباب غير الملتحقين بالتعليم أو التوظيف أو التدريب كنسبة مئوية من إجمالي عدد الثباب، أى يعتبر مقياسًا للشباب الذين هم خارج النظام التعليمي، وليسوا في التدريب ولا يمارسون أى عمل، وبالتالي فهو بمثابة مقياس أوسع للداخلين المحتملين لسوق عمل الثباب من بطالة الثباب، لأنه يشمل أيضًا الثباب خارج القوة العاملة وليسوا في التعليم أو التدريب، كما يعد هذا المؤشر أيضًا مقياسًا أفضل للوضع الحالي للداخلين المحتملين من الثباب إلى سوق العمل مقارنة بمعدل عدم نشاط الشباب، حيث يثمل الأخير هؤلاء الثباب الذين ليسوا في القوى العاملة ولكنهم مازالوا في التعليم، وبالتالي لا يمكن اعتبارهم متاحين حاليًا عمل، يتبين من جدول (r) تحسن الوضع بالنسبة لأفريقيا من حيث تلك النسبة على مستوى الاجمالى خلال تلك الفترة مقارنة بالنسبة للعالم. 


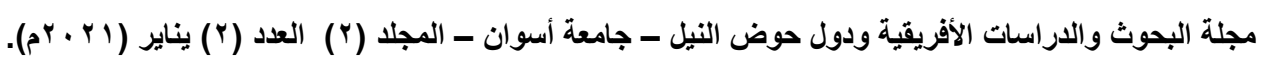

جدول (ץ) معدل فقر العمالة (النسبة المئوية للعاملين الذين يعيشون بأقل من • 1.9 دولار

\begin{tabular}{|c|c|c|c|c|c|c|c|c|c|c|}
\hline $\begin{array}{c}r \leqslant-10 \\
\text { عامL }\end{array}$ & فأكثر عام & السنة & النوع & المنطقة & فأكثر & $\begin{array}{c}r \text { ع } 10 \\
\text { عامL }\end{array}$ & فأكثر عام & السنة & النوع & المنطقة \\
\hline 44.0 & 37.8 & 2010 & الاجمالى & أفريقيا & 13.2 & 19.8 & 14.3 & 2010 & الاجمالى & العالم \\
\hline 42.0 & 34.4 & 2010 & الرجال & أفريقيا & 13.0 & 19.6 & 14.1 & 2010 & الرجال & العالم \\
\hline 46.4 & 42.4 & 2010 & النساء & أفريقيا & 13.4 & 20.1 & 14.5 & 2010 & النساء & العالم \\
\hline 43.2 & 36.8 & 2011 & الاجمالى & أفريقيا & 11.4 & 17.7 & 12.4 & 2011 & الاجمالى & العالم \\
\hline 41.6 & 33.5 & 2011 & الرجال & أفريقيا & 11.2 & 17.6 & 12.2 & 2011 & الرجال & العالم \\
\hline 45.2 & 41.2 & 2011 & النساء & أفريقِيا & 11.6 & 18.0 & 12.6 & 2011 & النساء & العالم \\
\hline 42.1 & 35.4 & 2012 & الاجمالى & أفريقبا & 10.1 & 16.3 & 11.0 & 2012 & الاجمالى & العالم \\
\hline 40.7 & 32.2 & 2012 & الرجال & أفريقِيا & 9.9 & 16.1 & 10.8 & 2012 & الرجال & العالم \\
\hline 43.7 & 39.6 & 2012 & النساء & أفريقيا & 10.4 & 16.6 & 11.4 & 2012 & النساء & العالم \\
\hline 41.4 & 34.6 & 2013 & الاجمالى & أفريقيا & 8.4 & 14.3 & 9.3 & 2013 & الاجمالى & العالم \\
\hline 40.1 & 31.5 & 2013 & الرجال & أفريقيا & 8.4 & 14.2 & 9.3 & 2013 & الرجال & العالم \\
\hline 42.9 & 38.7 & 2013 & النساء & أفريقيا & 8.4 & 14.4 & 9.3 & 2013 & النساء & العالم \\
\hline 41.0 & 33.9 & 2014 & الاجمالى & أفريقيا & 7.9 & 14.0 & 8.8 & 2014 & الاجمالى & العالم \\
\hline 39.9 & 30.9 & 2014 & الرجال & أفريقيا & 7.9 & 13.9 & 8.7 & 2014 & الرجال & العالم \\
\hline 42.2 & 37.9 & 2014 & النساء & أفريقيا & 7.9 & 14.1 & 8.8 & 2014 & النساء & العالم \\
\hline 40.7 & 33.4 & 2015 & الاجمالى & أفريقيا & 7.4 & 13.7 & 8.3 & 2015 & الاجمالى & العالم \\
\hline 40.0 & 30.5 & 2015 & الرجال & أفريقيا & 7.4 & 13.6 & 8.3 & 2015 & الرجال & العالم \\
\hline 41.6 & 37.3 & 2015 & النساء & أفريقيا & 7.5 & 13.9 & 8.4 & 2015 & النساء & العالم \\
\hline 40.5 & 33.0 & 2016 & الاجمالى & أفريقيا & 7.1 & 13.7 & 8.0 & 2016 & الاجمالى & العالم \\
\hline 39.9 & 30.1 & 2016 & الرجال & أفريقيا & 7.1 & 13.5 & 8.0 & 2016 & الرجال & العالم \\
\hline 41.3 & 36.9 & 2016 & النساء & أفريقيا & 7.3 & 13.9 & 8.2 & 2016 & النساء & العالم \\
\hline 39.9 & 32.4 & 2017 & الاجمالى & أفريقيا & 6.8 & 13.4 & 7.7 & 2017 & الاجمالى & العالم \\
\hline 39.4 & 29.6 & 2017 & الرجال & أفريقيا & 6.7 & 13.2 & 7.6 & 2017 & الرجال & العالم \\
\hline 40.6 & 36.1 & 2017 & النساء & أفريقيا & 7.1 & 13.7 & 7.9 & 2017 & النساء & العالم \\
\hline 39.2 & 31.6 & 2018 & الاجمالى & أفريقِا & 6.5 & 13.1 & 7.4 & 2018 & الاجمالى & العالم \\
\hline 38.7 & 28.9 & 2018 & الرجال & أفريقيا & 6.4 & 12.8 & 7.2 & 2018 & الرجال & العالم \\
\hline 39.7 & 35.2 & 2018 & النساء & أفريقيا & 6.8 & 13.5 & 7.7 & 2018 & النساء & العالم \\
\hline 38.4 & 30.9 & 2019 & الاجمالى & أفريقبا & 6.3 & 12.8 & 7.1 & 2019 & الاجمالى & العالم \\
\hline 38.0 & 28.2 & 2019 & الرجال & أفريقيا & 6.1 & 12.5 & 6.9 & 2019 & الرجال & العالم \\
\hline 38.9 & 34.4 & 2019 & النساء & أفريقِا & 6.6 & 13.3 & 7.5 & 2019 & النساء & العالم \\
\hline 37.6 & 30.2 & 2020 & الاجمالى & أفريقِا & 6.0 & 12.6 & 6.9 & 2020 & الاجمالى & العالم \\
\hline 37.2 & 27.6 & 2020 & الرجال & أفريقيا & 5.8 & 12.2 & 6.6 & 2020 & الرجال & العالم \\
\hline 38.1 & 33.6 & 2020 & النساء & أفريقبا & 6.4 & 13.2 & 7.3 & 2020 & النساء & العالم \\
\hline 36.9 & 29.5 & 2021 & الاجمالى & أفريقيا & 5.8 & 12.4 & 6.6 & 2021 & الاجمالى & العالم \\
\hline 36.5 & 26.9 & 2021 & الرجال & أفريقيا & 5.5 & 12.0 & 6.3 & 2021 & الرجال & العالم \\
\hline 37.3 & 32.7 & 2021 & النساء & أفريقِا & 6.3 & 13.0 & 7.1 & 2021 & النساء & العالم \\
\hline 36.2 & 28.8 & 2022 & الاجمالى & أفريقيا & 5.6 & 12.2 & 6.4 & 2022 & الاجمالى & العالم \\
\hline 35.8 & 26.3 & 2022 & الرجال & أفريقيا & 5.3 & 11.8 & 6.1 & 2022 & الرجال & العالم \\
\hline 36.5 & 31.9 & 2022 & النساء & أفريقيا & 6.1 & 12.9 & 7.0 & 2022 & النساء & العالم \\
\hline 35.4 & 28.1 & 2023 & الاجمالى & أفريقيا & 5.4 & 12.0 & 6.3 & 2023 & الاجمالى & العالم \\
\hline
\end{tabular}




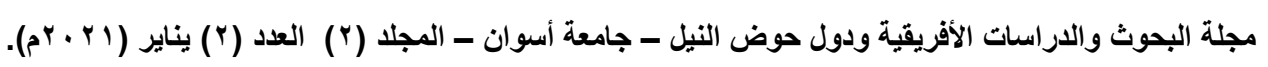

\begin{tabular}{|c|c|c|c|c|c|c|c|c|c|c|}
\hline 35.2 & 25.7 & 2023 & الرجال & أفريقيا & 5.1 & 11.6 & 5.9 & 2023 & الرجال & العالم \\
\hline 35.8 & 31.1 & 2023 & النساء & أفريقيا & 6.0 & 12.8 & 6.8 & 2023 & النساء & العالم \\
\hline 34.7 & 27.4 & 2024 & الاجمالى & أفريقيا & 5.3 & 11.9 & 6.1 & 2024 & الاجمالى & العالم \\
\hline 34.5 & 25.1 & 2024 & الرجال & أفريقيا & 4.9 & 11.4 & 5.7 & 2024 & الرجال & العالم \\
\hline 35.0 & 30.3 & 2024 & النساء & أفريقيا & 5.9 & 12.6 & 6.7 & 2024 & النساء & العالم \\
\hline
\end{tabular}

المصدر:

- International Labour Organization, Statistics and database, labour market indicators.

جدول (ץ) نسبة الثباب (الذين تتراوح أعمارهم بين 10 - ؛ ع عامًا) غير الملتحقين بالتعليم أو

العمل أو التدريب البنابه

\begin{tabular}{|c|c|c|c|c|c|c|c|c|c|}
\hline النساء & الرجال & الاجمالى & السنة & المنطقة & النساء & الرجال & الاجمالى & السنة & المنطقة \\
\hline 27.3 & 14.5 & 20.9 & 2010 & أفربقيا & 31.8 & 12.8 & 22.0 & 2010 & العالم \\
\hline 26.8 & 14.7 & 20.7 & 2011 & أفربقيا & 31.5 & 12.6 & 21.8 & 2011 & العالم \\
\hline 26.3 & 14.6 & 20.4 & 2012 & أفربقيا & 31.4 & 12.5 & 21.7 & 2012 & العالم \\
\hline 25.9 & 14.3 & 20.0 & 2013 & أفربقيا & 31.5 & 12.8 & 21.9 & 2013 & العالم \\
\hline 25.7 & 14.4 & 20.0 & 2014 & أفربقيا & 31.2 & 12.8 & 21.7 & 2014 & العالم \\
\hline 25.4 & 14.7 & 20.0 & 2015 & أفربقيا & 31.0 & 12.9 & 21.7 & 2015 & العالم \\
\hline 25.5 & 15.0 & 20.2 & 2016 & أفربقيا & 30.8 & 13.1 & 21.7 & 2016 & العالم \\
\hline 25.5 & 15.2 & 20.3 & 2017 & أفربقيا & 30.8 & 13.3 & 21.8 & 2017 & العالم \\
\hline 25.6 & 15.5 & 20.5 & 2018 & أفربقيا & 30.9 & 13.6 & 22.0 & 2018 & العالم \\
\hline 25.7 & 15.7 & 20.7 & 2019 & أفربقيا & 31.1 & 13.9 & 22.2 & 2019 & العالم \\
\hline
\end{tabular}

source :International Labor Organization, Statistics and database, labor market indicators.

دـ الناتج لكل عامل (الناتج المحلي الإجمالي بالدولار بأساس عام I I ب)

تم حساب هذا المقياس لإنتاجية العمل باستخدام بيانات حول الناتج المحلي

الإجمالي (بالدولار الدولي بأساس عام (1 ـ ب) من خلال قاعدة بيانات مؤشرات التتمية

العالمية للبنك الدولي، وذلك لحساب إنتاجية العمل كناتج محلي إجمالي لكل عامل

بالإعتماد على استخدام تقديرات منظمة العمل الدولية لإجمالي العمالة، ويتبين من

جدول (ع) تزايد إنتاجية العامل بأفريقيا خلال الفترة المختارة والتى تزايدت من نحو 


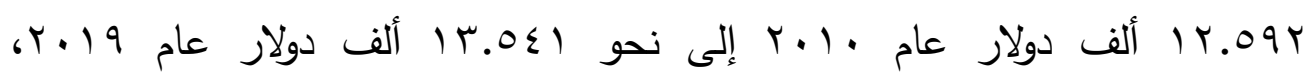
وبمتوسط بلغ نحو ..r.r ألف دولار خلال تلك الفترة، والذى يعكس أنه بإكتساب العامل للمهارات التى يتطلبها العمل من خلال التعليم والتدريب وإستخدام التكنولوجيا الحديثة ساهم ذلك فى إرتفاع إنتاجيته ومن ثم أجره (دخله) والذى ينعكس على مستوى الناتج المحلى للدولة ومن ثم معدل النمو الاقتصادى بها.

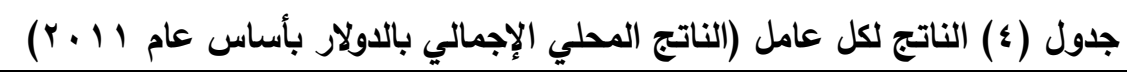

\begin{tabular}{|c|c|c|}
\hline أفربقيا & العالم & السنة \\
\hline 12592 & 30726 & 2010 \\
\hline 12493 & 31619 & 2011 \\
\hline 12982 & 32329 & 2012 \\
\hline 13166 & 33103 & 2013 \\
\hline 13339 & 33860 & 2014 \\
\hline 13427 & 34606 & 2015 \\
\hline 13402 & 35377 & 2016 \\
\hline 13509 & 36250 & 2017 \\
\hline 13555 & 37064 & 2018 \\
\hline 13541 & 37782 & 2019 \\
\hline 13200 & 34272 & المتوسط \\
\hline
\end{tabular}

source :International Labor Organization, Statistics and database, labor market indicators.

\section{• حالة مصر كنموذج رائد فى التعليم ما قبل الجامعى فى أفريقيا} تتتاولت هذه الجزئية من هذا الفصل إستعراض حالة مصر فى مجال التعليم قبل الجامعى فى قارة أفريقيا كمثال للنماذج الرائدة فى القارة الأفريقية فى التعليم قبل الجامعى. - التعليم قبل الجامعى فى مصر : 
يعد نظام التعليم فى مصر واحدا من أكبر نظم التعليم فى العالم، والأكبر على مستوى منطقة الثرق الأوسط وشمال أفريقيا، حيث يضم حوالى ع.. ب مليون طالب مزعين على مراحل التعليم المختلفة، تستحوذ مرحلة التعليم قبل الجامعى على نحو 1 1 مليون طالب أى ما يمثل حوالى ^^٪، فى حين يتم إستيعاب النسبة المتبقية فى مؤسسات التعليم العالى المختلفة' إنتهجت الدولة المصرية فى السنوات الأخيرة مسارا أسمته "التعليم المشروع القومى لمصر" ومن ثم وضعت وزارة التربية والتعليم خطة

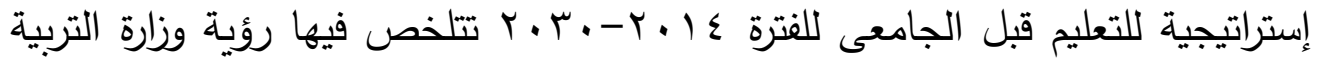
والتعليم في توفير موارد بشرية متنامية القدرة والكفاءة، وعلى أعلى درجة من الجودة والأخلاقيات المهنية، من أجل بناء مجتمع يقوم على التعلم واقتصاد يقوم على المعرفة. ولتحقيق هذه الرؤية تضطلع الوزارة برسالة قيادة وإدارة وتتمية قطاع التعليم قبل الجامعي ليستجيب للاحتياجات الاجتماعية والاقتصادية والثقافية للمجتمع المصري بهوية وطنية لا تنفصل عن الاتجاهات العالمية. وبذلك أصبح الهدف البعيد للقطاع هو التنمية الثاملة للنشء، مع غرس روح المواطنة والتسامح، ونبذ العنف، وتفهم أسس الحرية والعدالة من حقوق وواجبات و شعور بالمسؤولية تجاه الوطن والمواطنين. أما الهدف المباشر فيتمثل في التأكيد على الإلتزام بحق كل طفل في فرصة متكافئة لتلقي ' فتحى السيد يوسف عبدالمجيد (دكتور)، نظام التعليم المصرى: تقييم الوضع الحالى ومقترحات

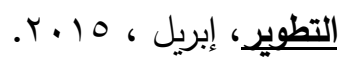


خدمة تعليمية بمستوى من الجودة يتتاسب مع المعايير العالمية، بما يسمح له بالإسهام الفعال في التتمية الاجتماعية والاقتصادية لبلده، وبالمنافسة إقليمياً وعالميا. ولتحقيق ما سبق تم تبنى ثلاث سياسات للإصلاح والتحسين تتفق وميثاق

\section{الأمم المتحدة لحقوق الإنسان، من خلال:}

ا- إتاحة فرص متكافئة لجميع السكان في سن التعليم للالتحاق وإكمال التعليم على مستوى فرعيه العام والفنى مع استهداف المناطق الفقيرة كأولوية أولى. r- تحسين جودة فعالية الخدمة التعليمية، من خلال توفير منهج معاصر، وتكنولوجيا موظفة بكفاءة، وأنشطة تربوية رياضية وغير رياضية، ومعلم فعال لكل طفل في كل فصل، وقيادة فعالة في كل مدرسة، وفرص للتنمية المهنية الداخلية والخارجية لكل معلم وإداري ليتقدم ويتميز · ب- تدعيم البنية المؤسسية وخاصة في المدارس الفنية، وبناء قدرة العاملين بالتعليم على تطبيق اللامركزية على وجه يضمن الحوكمة الرشيدة.. الواضع الراهن للتعليم قبل الجامعي فى مصر: يكفل الدستور المصري حق التعليم المجاني الإلزامي لكل الأطفال المصرين من سن 7 إلى 10 عام. وتتص التشريعات على أن "تعليم الطفل يهدف إلى تكوينه علميا وثقافيا وروحيا وتتمية شخصيته ومواهبه وقدراته العقلية والبدنية إلى أقصى إمكاناتها، بقصد إعداد الإنسان المؤمن بوطنه وبقيم الخير والحق والإنسانية وتزويده 
بالقيم والدراسات النظرية والتطبيقية والمقومات التي تحقق إنسانيته وكرامته وتساعده على تحقيق ذاته وانتمائه لوطنه والإسهام بكفاءة في مجالات الإنتاج والخدمات لاستكمال التعليم العالي، وذلك على أساس من تكافؤ الفرص".

- - السلم التعليمي المصري: ينقسم السلم التعليمي المصري إلى مرحلة تعليم أساسي وتتشكل من حلقتي التعليم الابتدائي والتعليم الإعدادي و مرحلة تعليم ثانوي، يستغرق التعليم الأساسي أعوام وتتص المادة (• ؟) من قانون التعليم على أن التعليم الأساسي يهدف إلى تتمية قدرات واستعدادات التلاميذ و إثباع ميولهم وتزويدهم بالقدر الضروري من القيم والسلوكيات والمعارف والمهارات العلمية والمهنية التي تتفق وظروف بيئاتهم المختلفة. بحيث يمكن لمن يتم مرحلة التعليم الأساسي أن يواصل تعليمه في مرحلة أعلى وأن يواجه الحياة بعد تدريب مهني مناسب، وذلك من أجل إعداد الفرد لكي يكون مواطن" منتجا في بيئته ومجتمعه. وينقسم التعليم الأساسي إلى حلتين، الابتدائية ومدتها ست سنوات وهي تقابل المستوى الأول (إسكد-1) وفقا للتصنيف الدولي لنظم التعليم، وهي تناظر المرحلة العمرية من 1 إلى ال سنة، ولتحق التلميذ بحلقة التعليم الابتدائى في سن 1 إلى ^ سنوات، حيث إن عمر ست سنوات يثكل العمر الرسمي للالتحاق بالتعليم. أما الحلقة الثانية من التعليم الأساسي فهي الحلقة الإعدادية ومدتها ثلاث سنوات، وهي تتاظر المرحلة العمرية من 12- 14سنة، وهي تقابل المستوى الثاني من 
التصنيف الدولي (إسكد-r) وتعمل الحلقة الإعدادية على تأهيل الطفل للالتحاق بالمرحلة الثانوية ، والتي تمثل المستوى الثالث (إسكد-r) بفرعية الأول والثالث، إذ إن المسار الأول من هذا المستوى وهو يعرف بالثانوي العام ومدته ثلاث سنوات يعد الطالب للالتحاق بالتعليم الجامعي أو بالتعليم ما بعد الثانوي ودون الجامعي (معاهد إعداد الفنيين) أما المسار الثاني من التعليم الثانوي فهو يعرف بالثانوي الفني ومدته من ثلاث إلى خمس سنوات ويعد الطالب للالتحاق بسوق العمل، ويتحدد قبول الطلاب بأي من المسارين على أساس الأداء في اختبار نهاية الحلقة الإعدادية وعلى رغبة الطالب والأماكن المتاحة بكل من المسارين. ويناظر التعليم الثانوي المرحلة العمرية من 15-17 سنة، وقد أضاف دستور (ع ( ب) المرحلة الثانوية إلى التعليم الإلزامي، ومن ثم أصبح التعليم الإلزامي يضم التعليم الأساسي والتعليم الثانوي بثقيه العام والفني. أما الأطفال الذين تجاوزوا سن الالتحاق بالتعليم الابتدائي (ثمان سنوات،) أو تسربوا منها، فيتم توجيهـم للالتحاق بمدارس الفصل الواحد أو مدارس المجتمع وهي مدارس حكومية تابعة لنظام التعليم العام أنشئ في التسعينيات وتعرف بمدارس الفرصة الثانية بمساعدة منظمة "يونيسف" ووزارة التربية والتعليم والمجتمع المحلي. ويتم افتتاحها في الغالب في المناطق محدودة السكان المحرومة من المدارس والمؤسسات التعليمية، وتتميز بنظام مرن يسمح بتدريس أكثر من مستوى في نفس الفصل. هنالك 
أشكال مختلفة من هذه المدارس التي تم تطويرها كي تستجيب إلى حاجات محددة مرتبطة بالمجتمع المحلي أو بالوضع الاجتماعي أوالجغرافي، وتقدم مدارس المجتمع في المناطق الريفية المحرومة مستوى تعليميا معادلا لحلقة التعليم الابتدائي، وهناك المدارس الصديقة للفتيات (مدارس الفصل الواحد) الهدف منها هو تقديم تعليم ذى جودة عالية ومناسب ومرن، تستهدف هذه المدارس بشكل أساسي البنات اللائي لم يلتحقن أو لم يرغبن في الالتحاق بالتعليم الابتدائى أو اللاتي تسربن من التعليم، وتكيف المدارس نفسها مع الضروريات المحلية وتقبل البنات في سن من 7 إلى ع عاما، والأولوية تكون للأطفال الأكبر سنا وخصوصا البنات، ويسح بالتحاق الذكور

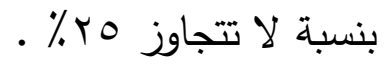

ويقدم قطاع التعليم في مصر برنامجا لمرحلة رياض الأطفال للمرحلة العمرية

4-5 سنوات وهو يعادل المستوى (إسكد-·) في التصنيف الدولي ولكنه ليس جزءا أساسيا من السلم التعليمي أو من التعليم الإلزامي، بمعنى أن الانتظام في هذه المرحلة لا يعتبر شرطًا مسبقا للقبول بالمرحلة الابتدائية.

• كما تهذف استراتيجية إصلاح التعليم في وزارة التربية والتعليم والتعليم الفني بمصر إلى تحسين مخرجات التعلم من خلال محورين'

${ }^{1}$ Understanding Middle East Education, Egypt Country Profile, PwC Education and Skills Practice, First Edition, 2018/2019. 
1- المحور الأول: والذي يتضمن مجموعة من المبادرات التي تهدف إلى تعديل وإصلاح نظام التعليم الحالي هيكليًا.

r- المحور الثانى: والذي يتضمن مجموعة من التخخلات الجادة التي تهدف إلى تحسين النتائج التعليمية وتحديث التعليم لتحقيق أهداف رؤية مصر ـ • r. وبرنامج الإصلاح الذي بدأ تنفيذه في العام الدراسي الذي بدأ في سبتمبر ^| • ؟، يتبنى التكنولوجيا الحديثة للتدري والتعلم وتقييم الطلاب وجمع البيانات، وتتضمن بعض التدخلات ذات الصلة ما يلي: أ-بنك المعرفة المصري: أرشيف مكتبة على الإنترنت يمكن الوصول إليه بواسطة الهوية الوطنية ويوفر خدمات تعليمية، الموارد البحثية، والثقافية لجميع المواطنين. ب- التعلم القائم على التكنولوجيا: توزيع مليون جهاز لوحي على الصعيد الوطني بين طلاب الصف العاشر والمعلمين والمدرسين المدبرين مع المحتوى ذي الصلة لتحسين المشاركة التعليمية. ت-تحديث تقييم الطالب: غيّر برنامج الإصلاح طريقة التقييم للددرسة الثانوية الوطنية (الثانوية العامة)، التي تحدد مسارات التعليم التي سيتبعها الطلاب - كانت امتحانات نهاية العام فى 12-G10 استبداله بمعدل تراكمي ץ سنوات، لتجنب أي 
تسرب محتمل لأسئلة الامتحان، سيتم استبدال الاختبارات الورقية بـ الاختبارات الإلكترونية التي تعمل على تحسين وتوحيد تقييم الطلاب. - مجال زيادة المخصصات الخاصة لقطاع التعليم فى مصر تتمتع مصر بنظام تعليم عام مدعوم كما أنه في عام ع ا ـ. تم تعديل الدستور في مصر وزادت سنوات تمويل الدولة التعليم الإلزامي حتى نهاية المرحلة الثانوية أو ما يعادلها، وقد أدى ذلك إلى زيادة الإنفاق العام السنوي على تعليم حيث بلغ V• ا مليار

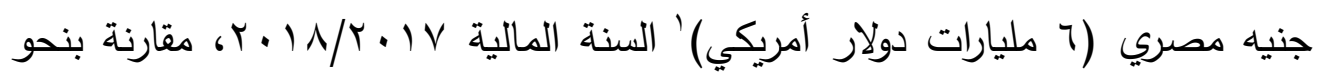

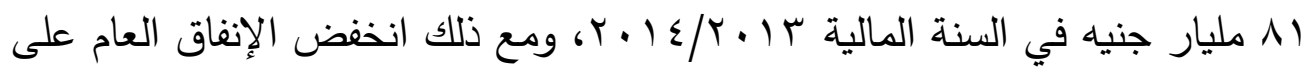
التعليم كنسبة مئوية من إجمالي الإنفاق ليمثل 9٪ فقط في السنة المالية

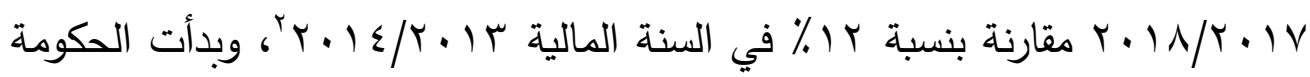
المصرية في تشجيع مشاركة القطاع الخاص والاستثمارات في قطاع التعليم للمساعدة

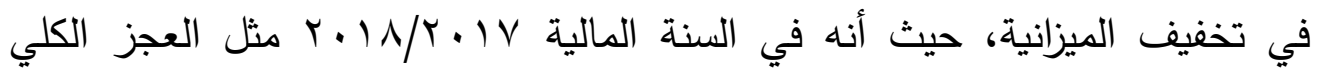
للموازنة حوالي • ـ من الناتج المحلي الإجمالي، كما بلغ إجمالي الدين العام 9 ـ 1٪

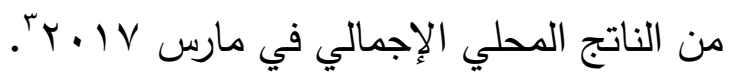

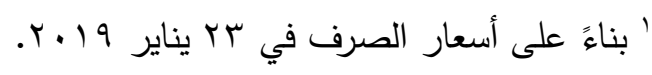

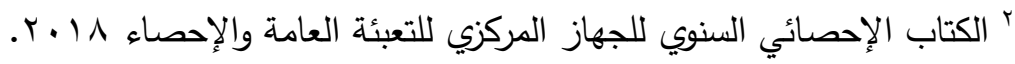

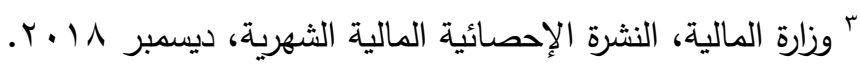


وتجدر الاشارة إلى أنه في الوقت الحالي يشكل القطاع الخاص • ( من إجمالي المسجلين في نظام التعليم K-12 في مصر، والمدارس الخاصة تستوعب ع \% من طلاب رياض الأطفال وحوالى ^^ من تلاميذ المدارس الابتدائية وحوالى \٪ من طلاب المرحلة الإعدادية وحوالى ب ا ٪ من العام طلاب المرحلة الثانوية'، مع ذلك فمن المتوقع أن يزداد مشاركة التعليم الخاص في مصر بتوفير دوافع الاستثمار المناسبة التالية: - n - n

1 - طلب مستدام على التعليم بسبب النمو السكاني المتزايد عدد سكان مصر من الثباب تزايد على مدى العقد الماضي، حيث نما عدد سكان مصر بشكل بمتوسط معدل سنوي بلغ حوالى ؟.Y\%، وفى عام Y . . كان 10 \% من المصريين دون سن 0ب عامًا وفقا لتوقعات بيانات الأمم المتحدة، هذا النمو سيضع مزيدا من الضغط على توفير ومشاركة التعليم الخاص. ץ - تحسين وضع الاقتصاد الكلي في محاولة لمعالجة الاختلالات الكبيرة في الاقتصاد الكلي التي واجهتها مصر منذ عام || ـ إستعادة الوضع المالي والاستقرار النقدي، حيث شرعت الحكومة في برنامج إصلاح اقتصادي شامل في نوفمبر Y 1 ـ r، كما أنه وافق صندوق النقد الدولي 
على حزمة مساعدات مالية لمصر بقيمة r ا مليار دولار ، بعض الإصلاحات المالية والنقدية الرئيسية فيما يلي توضيح للتدابير التي اتخذتها الحكومة المصرية. - الحاجة إلى الاستثمار في سد الفجوات القائمة في المهارات

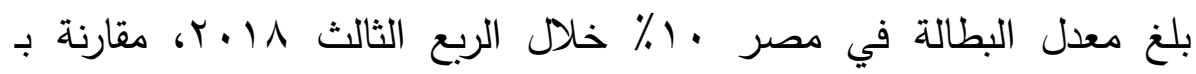

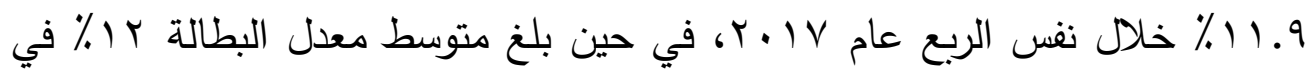

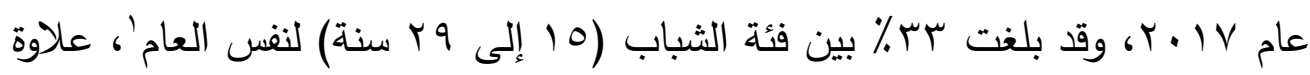

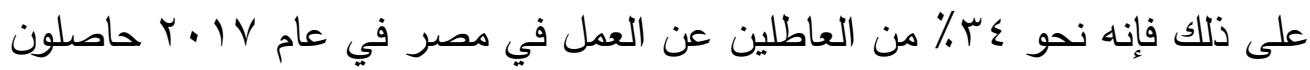
على درجات البكالوريوس أو الدراسات العليا، تمثل ثاني أكبر فئة بطالة حسب الحالة التعليمية، مما يشير إلى أن المعروض الخريجين لا يكتسبون المهارات المطلوبة من قبل أصحاب العمل الذين يقودون هذا النمو الاقتصادي. وقد أقرت الحكومة بمسؤوليتها عن ضمان حق كل طفل مصري في التعليم، وقد استلزم هذا التغيير السريع الانتقال نحو مجتمع المعرفة، وتحسين معالجة واستيعاب تحديات القرن الحادي والعشرين والسعي لتحقيق الأهداف الاستراتيجية التي أقرتها خطة

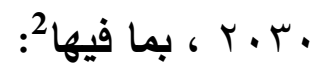

' ل ق قاعدة بيانات منظمة العمل الدولية، 2018.

2 Nermeen Singer, El-Farahaty El-Sayed Mahmoud, MOTIVES OF THE EGYPTIAN EDUCATION FUTURE FOR SUSTAINABLE DEVELOPMENT: A COMPARATIVE ANALYSIS BETWEEN 2020 AND 2030, Humanities \& Social Sciences Reviews, Vol 8, No 2e, April 2020. 
1- تتمية المهارات ضرورية للتعليم والمتعلمين ليصبحوا مواطنين ناجحين ومسؤولين واثثين من أنفسهم. ץ- توفير التعليم وفرص التعلم التي تعمل على احترام الحريات الأساسية لجميع شرائح المجتمع، وتدريب الطلاب على تحمل المسؤولية في مجتمع حر وفق روح التفاهم والسلام والتسامح والصداقة بين الجماعات القومية والدينية. r- تتمية احترام البيئة الطبيعية وتعظيم فرص التتمية الثقافية واحترامها على مستوى الأفراد والجماعات، وتتمية شخصية الطفل، والعبقرية الطبيعية والعقلية إلى أقصى حد. ع- تطوير وتتفيذ أنظمة المساءلة لتحسين الأداء ورفع كفاءة الموظفين لكسب ثقة الجمهور في جميع جوانب الخدمة لضمان التحسين المستمر في الأداء. ه- دعم تعلم التلاميذ وتجهيزهم للعيش في المجتمع المحلي والدولي حسب توظيف واستخدام تكنولوجيا المعلومات والاتصالات.

צ- تقويم الأطفال والثباب والتفكير في مهاراتهم وتحديد خطوات تحسين مهاراتهم وفهههم وكيف يمكن استخدام المهارات التي اكتسبوها في حياتهم داخل وخارج الفصل الدراسي أو المدرسة. V- توفير التعليم بالشراكة مع جميع المؤسسات والثركاء المعنيين بشأن تتمية المهارات وتوظيف. 
- الهدف الرابع من أهداف التنمية المستدامة بثأن التعليم "الجمعية العامة للأمم

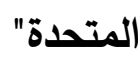
نص الهدف على "ضمان تعليم جيد وعادل وشامل وتعزيز فرص التعلم مدى

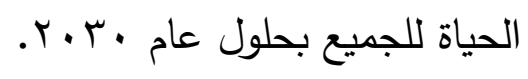

يرتبط هذا الهدف بالأهداف التالية التي يمكن تحقيقها بمصر بحلول عام

$$
: Y \cdot Y, 1
$$

• ضمان حصول جميع الفتيات والفتيان على تعليم ابتدائي وثانوي مجاني وعادل وعالي الجودة، مما يؤدي إلى تعليم مناسب ومخرجات تعليمية فعالة. التأكد من أن جميع الفتيات والفتيان يتمتعون بفرص ممتازة للتطور ورعاية الطفولة المبكرة والتعليم قبل المدرسي حتى يكونوا جاهزين للتعليم الابتدائي. • ضمان الوصول المتكافئ لجميع النساء والرجال إلى التعليم الفني والمهني وبجودة عالية وبأسعار معقولة التعليم. • ضمان زيادة كبيرة في عدد الثباب والبالغين ذوي المهارات المناسبة، بما في ذلك التقنية والمهارات المهنية للعمل والوظائف اللائقة وريادة الأعمال.

1 Nermeen Singer, El-Farahaty El-Sayed Mahmoud, MOTIVES OF THE EGYPTIAN EDUCATION FUTURE FOR SUSTAINABLE DEVELOPMENT: A COMPARATIVE ANALYSIS BETWEEN 2020 AND 2030, Humanities \& Social Sciences Reviews, op. 
معالجة التفاوتات بين الجنسين في التعليم وضمان الوصول إلى جميع مستويات التعليم والتدربب المهني، بما في ذلك الأشخاص ذوو الإعاقة والسكان الأصليون والأطفال في المواقف الخطرة. التأكد من أن جميع الثباب ونسبة كبيرة من البالغين، رجالاً ونساءً، يتعلمون القراءة والكتابة والحساب.

ضمان اكتساب جميع المتعلمين المعرفة والمهارات اللازمة لتعزيز التتمية المستدامة من خلال، التعليم وحقوق الإنسان، والمساواة بين الجنسين، وتعزيز ثقافة السلام واللاعنف والمواطنة العالمية وتقدير التنوع الثقافي (الأمم المتحدة ، 10 ـ ؟). بناء وتحديث المرافق التعليمية التي تراعي الفوارق بين الجنسين والإعاقة قيد التطوير للمرافق التعليمية وخلق بيئات تعلم منتجة وآمنة وغير عنيفة للجميع. زيادة عدد المنح الدراسية للتعليم العالي المتاحة للبلدان النامية، ولا سيما البلدان الأقل نموا، الدول الجزرية الصغيرة النامية، والدول الأفريقية، بما في ذلك منح للتدربب المهني، تكنولوجيا المعلومات والاتصالات والبرامج التقنية والهندية والعلمية في البلدان المتقدمة والبلدان النامية الأخرى. 
تحقيق زيادة كبيرة في عدد المعلمين المؤهلين من خلال التعاون الدولي للمعلمين والتدريب، ولا سيما البلدان الأقل نموا والدول الجزرية الصغيرة النامية (اللجنة الأوروبية

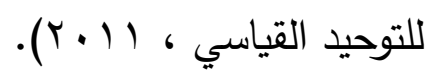

الهدف الرابع للتمية المستدامة يؤكد على دور التعليم الشامل والطموح في أجندة

التتمية المستقبلية من خلال تضمين أهداف رعاية الطفولة المبكرة والتعليم ذات الصلة بالمهارات من أجل الوظائف اللائقة، والتعليم من أجل التنمية المستدامة، والمواطنة العالمية (Kutesa 2015)، والتركيز على جودة التعليم، وهذا ليس بجديد لأن الهدف السادس وهو التعليم للجميع تتاول نوعية التعليم حيث أظهر أن المؤشر الكمي لمعدل استمرار التلاميذ حتى الصف الخامس يضعف التركيز على تحقيق الجودة الحقيقية، ونأمل أن يركز الهدف الرابع للتمية المستدامة على جودة التعليم والواقعية في خطط التتفيذ المستقبلية، تحديد المؤشرات المناسبة للتعجيل بتحسين جودة التعليم لا يزال بعيد المنال في الإطار المقترح (اليونسكو ، 10 • ب). يعد الوصول إلى التعليم الجيد على جميع المستويات شرطًا أساسيًا للتتمية المستدامة، والقضاء على الفقر، والمساواة بين الجنسين المساواة، وتمكين المرأة، والتتمية البشرية، والمشاركة الكاملة للمرأة والرجل على

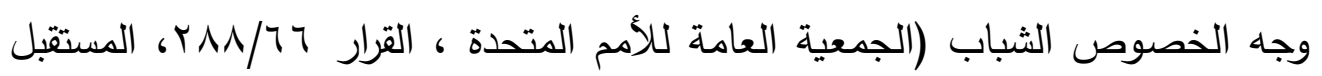

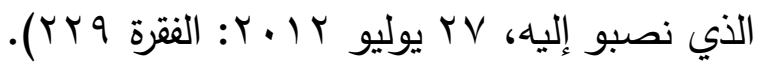


مجلة البحوث و الدراسات الأفريقية ودول حوض النيل - جامعة أسوان - المجلد (Y) العدد (Y) يناير ( Y • Y م). 


\section{الملخص}

إستهاف البحث بصفة أساسية دراسة وتوضيح تأثير التعليم والتدريب على

إقتصاد الدول الأفريقية، وكذا إلقاء الضوء على العلاقة بين التعليم والاقتصاد والتتمية كعلاقة تبادلية حيث يسهم التعليم في التتمية بشكل مباشر، وإستعرض البحث إستراتيجية التعليم القارية لأفريقيا والتى تتضمن مجموعة من الأهداف الاستراتيجية تتمثل فى إثنى عشر هدف تم إستعراضهم بمتن البحث، وكذا كانت أهم النتائج للمؤشرات التى تعكس أثر التعليم على النمو الاقتصادى بأفريقيا تتمثل فى الأتى: - معدل النمو السنوي للناتج لكل عامل والذى يظهر معدلات النمو السنوية لإنتاجية العمل، والذى يسمح بتقييم معدلات النمو فى انتاجية العمالة المساهمة فى الناتج المحلى الاجمالى بمرور الوقت، وبالتالي توفير معلومات حول كفاءة وجودة رأس المال البشري في عملية الإنتاج لوضع اقتصادي واجتماعي معين، بالاضافة لذلك المدخلات التكميلية الأخرى والابتكارات المستخدمة في الإنتاج، بلغ متوسط وحدة العمل خلال تلك الفترة حوالى ^. 1 ٪ بالعالم، فى حين بلغ حوالى 9 . . ؛ لكل عامل بأفريقيا أى ما يساوى نصف معدل الناتج لوحدة العمل على مستوى العالم، ويتسم معدل نمو إنتاجية العامل بأفريقيا خلال تلك الفترة بالتذبذب وعدم الاستقرار . - معدل فقر العمالة يبين هذا المعدل النسبة المئوية للأشخاص العاملين الذين يعيشون في فقر بالرغم من أنهم يعملون، ويتم تعريف الفقر باستخدام خط الفقر الدولي 
البالغ •9. 1 دولار أمريكي في اليوم، أى النسبة المئوية من العاملون الذين يتقاضون أقل من 1.9 بمرور الزمن على مستوى النوع (رجال، نساء)، وكذا على مستوى الفئات العمرية المختلفة القادرة على العمل والمشاركة فيه وهذا يبين أنه كلما زاد مستوى التعليم والالمام بالمعرفة والتكنولوجيا كلما كانت هناك وظائف أفضل وكذا الأجور أو المرتبات أفضل وبالتالى تتحسن مستويات المعيشة. - نسبة الشباب (الذين تتراوح أعمارهم بين 0 - 1- ع عامًا) غير الملتحقين بالتعليم أو العمل أو التدريب، ويعتبر مقياسًا للشباب الذين هم خارج النظام التعليمي، وليسوا في التدريب ولا يمارسون أى عمل، وبالتالي فهو بمثابة مقياس أوسع للداخلين المحتملين لسوق عمل الشباب من بطالة الشباب، لأنه يشمل أيضًا الشباب خارج القوة العاملة وليسوا في التعليم أو التدريب، كما يعد هذا المؤشر أيضًا مقياسًا أفضل للوضع الحالي للداخلين المحتملين من الثباب إلى سوق العمل مقارنة بمعدل عدم نشاط الثباب، حيث يشمل الأخير هؤلاء الثباب الذين ليسوا في القوى العاملة ولكنهم مازالوا في التعليم، وبالتالي لا يمكن اعتبارهم متاحين حاليًا عمل، وقد تبين تحسن الوضع بالنسبة لأفريقيا من حيث تلك النسبة على مستوى الاجمالى خلال تلك الفترة مقارنة بالنسبة للعالم. 
- وعلى مستوى الناتج لكل عامل تبين تزايد إنتاجية العامل بأفريقيا خلال الفترة

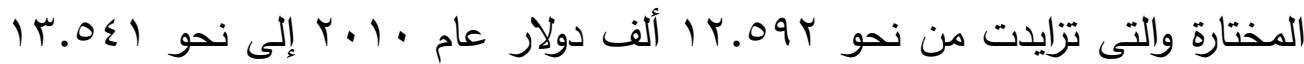
ألف دولار عام 19 •.Y، وبمتوسط بلغ نحو ....r. ألف دولار خلال تلك الفترة، والذى يعكس أنه بإكتساب العامل للمهارات التى يتطلبها العمل من خلال التعليم والتدريب وإستخدام التكنولوجيا الحديثة ساهم ذلك فى إرتفاع إنتاجيته ومن ثم أجره (دخله) والذى ينعكس على مستوى الناتج المحلى للدولة ومن ثم معدل النمو

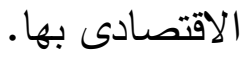

المراجح:

( ) عبدالله ونيس الترهونى (دكتور)، العلاقة بين النمو الاقتصادى وجودة التعليم، بوابة أفريقيا

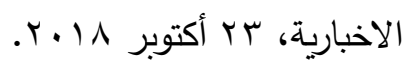

r) فتحى السيد يوسف عبدالمجيد (دكتور)، نظام التعليم المصرى: تقييم الوضع الحالى ومقترحات

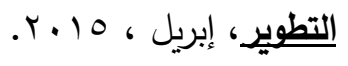

ץ قاعدة بيانات منظمة العمل الدولية، 2018.

ع) الكتاب الإحصائي السنوي للجهاز المركزي للتعبئة العامة والإحصاء 11 ـــ.

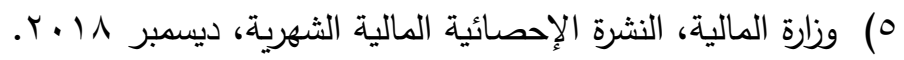

6) AFRICAN UNION, Continental Education Strategy for Africa, Indicators Manual, 2016-2025.

7) British Council (2014), "Can higher education solve Africa's job crisis? Understanding graduate employability in SubSaharan 


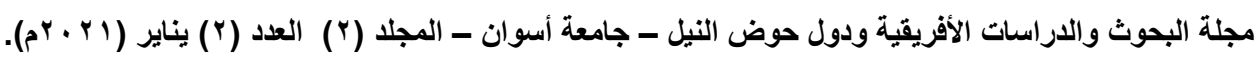

Africa, " available for download at: https://goo.gl/JEdTuj accessed on 19 July 2018.

8) Facing Forward: Schooling for Learning in Africa, worldbank, March 5, 2018.

9) https://www.almrsal.com/post/767576.

10) Masemula M.B, Whose Knowledge is Transmitted through Public Education in Africa?. In: Abdi A.A., Shultz L., Pillay T. eds Decolonizing Global Citizenship Education, 2015.

11) National Bureau of Statistics (2017),"- Labor Force Statistics Vol 1: Unemployment and Underemployment Report", available for download at:http://nigerianstat.gov.ng/download/694 accessed on 19 July, 2018.

12) Nermeen Singer, El-Farahaty El-Sayed Mahmoud, MOTIVES OF THE EGYPTIAN EDUCATION FUTURE FOR SUSTAINABLE DEVELOPMENT: A COMPARATIVE ANALYSIS BETWEEN 2020 AND 2030, Humanities \& Social Sciences Reviews, Vol 8, No 2e, April 2020.

13) Nwanosike, O. F \& ,.Onyije, F, Colonialism and education. Mediterranean journal of social sciences, $2(4), r \cdot 1)$.

14) Quartz Africa (2016). University degrees are not the answer for Africa's unemployed youth, Available online https://goo.gl/gN6f39 accessed on 20 July, 2018.

15) Rodney, W. How europe underdeveloped Africa. Pambazuka Press, 2012.

16) Understanding Middle East Education, Egypt Country Profile, PwC Education and Skills Practice, First Edition, 2018/2019. 